Background Hepatocellular carcinoma (HCC) has become a prominent global health threat due to its occurrence, lethality and dismal survival rates. Increasing prevalence of obesity and diabetes-induced non-alcoholic fatty liver disease (NAFLD) and metabolic syndromes have been found culpable for the rise of HCC initiation, via disruption of liver microenvironment. Super enhancers, which are characterised by high density of transcription binding sites, high-level transcription regulation and response to external stimulation, determine cell fate during oncogenesis. Master transcription factors translate microenvironmental changes into super enhancer remodelling and activation, which subsequently changes the gene expression profile and define cell identity. This project aims at profiling the super enhancer status in the context of NAFLD-associated HCC and to unveil the master transcription factors responsible for dietinduced HCC progression.

Methods Nanoscale chromatin immunoprecipitation sequencing (nano ChIP-seq) against histone marks H3K27ac, H3K4me1 and $\mathrm{H} 3 \mathrm{~K} 4 \mathrm{me} 3$ in 6 pairs of primary human NAFLD-HCC tumours and their adjacent non-tumour tissues revealed potential oncogenic super enhancers. Global mRNA expression was detected by RNA sequencing (RNA-seq) to support the enhancer-target gene transcription axis. Master transcription factor regulation of NAFLD-HCC super-enhancers was further supported by integrated bioinformatics analysis, including motif enrichment and signature transcription factor discovery. ChIP-seq data for the master transcription factors in HepG2 cells confirmed their occupancies on super enhancers controlling key oncogenic pathways.

Results Tumor-enabling super enhancers (averaged 553 and 484 per HCC tumour and non-tumour tissue, respectively) were profiled in primary human NAFLD-HCC tissues, and master transcription factors showed significant binding to NAFLD-HCC oncogenic super enhancers. Interestingly, tumorenabling super enhancers co-bound by HCC-specific master transcription factors target critical genes involved in hepatic inflammation and NAFLD pathogenesis.

Conclusions Integrated analysis of chromatin profiling and transcriptome in primary human tissues provides insights into the trans-regulatory network involving oncogenic super enhancers and master transcription factors during the pathogenic process of metabolic syndrome-associated HCC.

Acknowledgement This work is supported by the RGC CRF (C4017-14G).

\section{IDDF2018-ABS-0229 ENHANCING THE EFFICACY OF LIVER CANCER IMMUNOTHERAPY BY SPECIFIC INHIBITION OF HISTONE DEACETYLASE 8}

${ }^{1}$ Yu Feng*, ${ }^{1}$ Weiqin Yang, ${ }^{1}$ Jingying Zhou, ${ }^{2}$ Hanyong Sun, ${ }^{2}$ Stephen L Chan, ${ }^{3}$ Zhiwei Chen, ${ }^{4}$ Ka-Fai To, ${ }^{1}$ Alfred Sze-Lok Cheng. ${ }^{1}$ School of Biomedical Sciences, The Chinese University of Hong Kong, Hong Kong; 'Department of Medicine and Therapeutics, The Chinese University of Hong Kong, Hong Kong; ${ }^{3}$ AIDS Institute, The University of Hong Kong, Hong Kong; ${ }^{4}$ Therese Chow Centre for Gastrointestinal Cancer, The Chinese University of Hong Kong, Hong Kong

\subsection{6/gutjnl-2018-IDDFbestabstracts.8}

Background Recent clinical trials of inhibition of immune-checkpoints, programmed death-ligand 1 (PD-L1)/PD-1 axis, which elicits anti-tumour T-cell responses in various cancers, have also produced durable efficacy in a fraction of patients with advanced hepatocellular carcinoma (HCC). The heterogeneous responses to immune-checkpoint blockade therapy result from the complicated interplay between cancer-cell-autonomous cues and the immunosuppressive tumour microenvironment. Our previous epigenomics and functional analysis have elucidated an oncogenic role of histone deacetylase 8 (HDAC8) in hepatic carcinogenesis (Cancer Research 2015;75:4803-16). In this study, we aimed to investigate the therapeutic potential of an HDAC8-specific inhibitor PCI-34051 in preclinical HCC model.

Methods To investigate the immune-modulatory and antitumour effects of PCI-34051, we established an orthotopic HCC mouse model via intrahepatic implantation of syngeneic Hepa1-6 hepatoma cells in immunocompetent C57BL/6 and immunodeficient nude mice. The mice have been treated with vehicle, PCI-34051, anti-PD-L1 antibody, or combined therapy for 2 weeks. The tumorigenicity was assessed by in vivo imaging and correlated with immune profiling.

Results We demonstrated that PCI-34051 significantly reduced HCC tumorigenicity in C57BL/6 $(\mathrm{p}<0.01)$ but not nude mice. Immune profiling revealed a specific reduction in tumor-infiltrating regulatory $\mathrm{T}$ cells (Tregs; $\mathrm{p}<0.05$ ), associated with significant increase in $\mathrm{CD} 8+\mathrm{T}$ cells $(\mathrm{p}<0.05)$. The functional significance of Tregs was demonstrated by adoptive transfer, which completely abrogated PCI-34051-induced tumour growth inhibition. Notably, PCI-34051 treatment significantly enhanced the efficacy of anti-PD-L1 therapy $(p<0.01)$. More importantly, combined PCI-34051 and anti-PD-L1 treatment resulted in complete tumour eradication in all co-treated mice, which exhibited significantly better survival rate than single treatment groups $(\mathrm{p}<0.05)$. Moreover, the combination therapy promoted long-term survival (>300 days), which was associated with elevated CD8+T memory cells.

Conclusions Our data suggest that selective chromatin modifications by HDAC8 alter the tumour immune surveillance program and demonstrate the potential of rational combinatorial epigenetic immunotherapy to fully unleash T-cell responses, leading to long-term remission of HCC.

\section{IDDF2018-ABS-0245 HONEY SUPPLEMENTATION AMELIORATES ADIPOCYTOKINES AND HEPATOTOXICITY INDUCED BY HIGH FAT DIET ON MALE RATS}

${ }^{1}$ Suhana Samat*, 'Francis Kanyan Enchang, 'Zolkapli Eshak, ${ }^{2}$ Wan Iryani Wan Ismail. ${ }^{1}$ Universiti Teknologi MARA (UITM), Puncak Alam Campus, Selangor, Malaysia; ${ }^{2}$ Universiti Malaysia Terengganu (UMT), Kuala Terengganu, Terengganu, Malaysia

\subsection{6/gutjin-2018-IDDFbestabstracts.9}

Background Nowadays, obesity has become a serious problem worldwide and the cause of many diseases. Honey has been used since the dawn of human civilization contains various ingredients that contribute to its medicinal properties and health-beneficial effects. For this reason, honey could be considered as a potential traditional remedy for various illnesses plaguing mankind. This study was conducted to investigate the protective role of honey supplementation in modulating the hepatic damage and obesity biomarkers associated with obesity on in vivo model.

Methods Animals were induced obesity by feeding high-fat diet for eight weeks. Then, they were divided into different groups which received normal diet (NC); fed with high-fat diet (HFD) alone or with the treatment of Acacia (HFDAH) or pineapple honey (HFDPH) or orlistat (HFDO). Before and after treatment, the blood samples were collected for assessment of adipocytokines biomarkers (adiponectin, resistin and leptin), lipid profiles (total cholesterol (TC), triglycerides (TG), 